\title{
SUBMICROSCOPIC PLASMODIUM FALCIPARUM GAMETOCYTE DENSITIES FREQUENTLY RESULT IN MOSQUITO INFECTION
}

\author{
PETRA SCHNEIDER,$\dagger$ J. TEUN BOUSEMA ${ }^{*} \dagger$ LOUIS C. GOUAGNA, SILAS OTIENO, \\ MARGA VAN DE VEGTE-BOLMER, SABAH A. OMAR, AND ROBERT W. SAUERWEIN \\ Department of Medical Microbiology, Radboud University Nijmegen Medical Centre, Nijmegen, The Netherlands; International \\ Centre for Insect Physiology and Ecology, Mbita, Kenya; Kenya Medical Research Institute, Nairobi, Kenya
}

\begin{abstract}
Submicroscopic Plasmodium falciparum gametocytemia $(<5,000$ gametocytes/mL) is common and may result in mosquito infection. We assessed the relation between gametocyte density and mosquito infection under experimental and field conditions using real-time quantitative nucleic acid sequence-based amplification (QT-NASBA) for gametocyte quantification. Serial dilutions of NF54 $P$. falciparum gametocytes showed a positive association between gametocyte density and the proportion of infected mosquitoes $(\beta=6.1 ; 95 \%$ confidence interval $[\mathrm{CI}], 2.7-9.6 ; P=$ 0.001). Successful infection became unlikely below an estimated density of 250-300 gametocytes/mL. In the field, blood samples of 100 naturally infected children showed a positive association between gametocyte density and oocyst counts in mosquitoes $(\beta=0.38 ; 95 \% \mathrm{CI}, 0.14-0.61 ; P=0.002)$. The relative contribution to malaria transmission was similar for carriers with submicroscopic and microscopic gametocytemia. Our results show that transmission occurs efficiently at submicroscopic gametocyte densities and that carriers harboring submicroscopic gametocytemia constitute a considerable proportion of the human infectious reservoir.
\end{abstract}

\section{INTRODUCTION}

Transmission of Plasmodium falciparum from human to mosquito depends on the presence of infectious sexual stage parasites (gametocytes) that are ingested by mosquitoes taking a blood meal. The likelihood of mosquito infection can be expected to increase proportionally with increasing gametocyte density. However, this relation remains equivocal. A positive relation between gametocyte density and the proportion of infected mosquitoes was detected in experimental ${ }^{1-3}$ and natural ${ }^{2,4-6} P$. falciparum infections, but this relation was weak or non-existing in other studies. ${ }^{7-11}$ Several studies show that mosquitoes can become infected with gametocyte densities below the detection level of standard microscopy. $3,6,8,9,12-14$ Importantly, the recent application of molecular techniques for the detection ${ }^{15,16}$ and quantification $^{17,18}$ of gametocytes indicated that submicroscopic gametocyte densities are common. ${ }^{17,19,20}$ In a previous study, we have shown that submicroscopic gametocyte densities seem sufficient to drive post-treatment malaria transmission. ${ }^{21}$ It is important to quantify this potential reservoir and to determine the relation between submicroscopic gametocyte densities and the infectiousness to mosquitoes to plan and evaluate transmission reducing interventions. ${ }^{22}$ This can be done in natural infections and under laboratory conditions where the use of cultured, synchronized gametocytes excludes potential confounders such as gametocyte maturity and host immune factors. In this study, we used quantitative nucleic acid sequence-based amplification (QT-NASBA) to determine the relation between $P$. falciparum gametocyte density and the infectiousness to mosquitoes under field and laboratory conditions.

\section{MATERIALS AND METHODS}

Infectiousness of cultured gametocytes. Plasmodium falciparum NF54 gametocytes were obtained from in vitro cul-

\footnotetext{
* Address correspondence to J. Teun Bousema, Department of Medical Microbiology 268, Radboud University, Nijmegen Medical Centre, PO Box 9101, 6500 HB Nijmegen, The Netherlands. E-mail: t.bousema@ncmls.ru.nl

$\dagger$ The first two authors each contributed equally to this manuscript.
}

ture. ${ }^{23}$ Experiments were performed with mature gametocytes. Serial dilutions were made in whole blood to obtain gametocyte dilutions at $\sim 50 \%$ hematocrit ranging from $10^{2}$ to $10^{6}$ gametocytes $/ \mathrm{mL}$. Gametocyte density in serially diluted suspensions was calculated from the number of gametocytes present in the original sample. Of all dilutions, a $50-\mu \mathrm{L}$ sample was stored at $-70^{\circ} \mathrm{C}$ for subsequent quantification of gametocytes by real-time $P f s 25$ QT-NASBA. All dilutions were fed through a membrane feeding system to 50-100 female Anopheles stephensi mosquitoes ${ }^{24}$ that were 3-5 days old and starved for 1-2 hours before feeding. After feeding, mosquitoes were kept at $26^{\circ} \mathrm{C}$ and $80 \%$ relative humidity on glucose until dissected in $1 \%$ mercurochrome for microscopical assessment of oocyst infection on days 7-9.

Infectiousness of naturally infected children. Membrane feedings were conducted on samples from children naturally infected with $P$. falciparum malaria in Mbita, Suba district, western Kenya. Details of the study can be found elsewhere. ${ }^{21}$ The study protocol was approved by the scientific steering committee and ethical review committee of the Kenya Medical Research Institute (SSC No. 791). Children were treated for uncomplicated $P$. falciparum malaria with sulphadoxinepyrimethamine (SP), SP plus artesunate, SP plus amodiaquine, or artemether-lumefantrine. All children older than 2 years who attended the clinic for their day 14 post-treatment visit in November-December 2004 were invited for membrane feeding experiments, regardless of microscopic gametocytemia. We enrolled 25 children consecutively per treatment arm, giving a total of 100 membrane feeding experiments.

Venous blood samples of $3 \mathrm{~mL}$ were obtained from children whose parent or guardian gave specific consent for the procedure. Samples were fed to $\sim 150$ locally reared 4 - to 5-day-old female Anopheles gambiae s.s. mosquitoes through an artificial membrane attached to a water-jacketed glass feeder maintained at $37^{\circ} \mathrm{C}$. After $10-15$ minutes, fully fed mosquitoes were selected and kept at $27-29^{\circ} \mathrm{C}$ for 7 days on glucose $(10 \%)$ until midguts were dissected in $2 \%$ mercurochrome. The midguts were examined microscopically for oocysts with a second microscopist confirming their presence if observed. For each experiment, exactly 30 randomly selected mosquitoes were dissected. From each blood sample included 
in the membrane feedings, $50 \mu \mathrm{L}$ was used for quantification of gametocytes by real-time $P f_{s} 25$ QT-NASBA.

Gametocyte detection by Pfs25 QT-NASBA. Quantification of gametocytes by real-time $P f s 25$ mRNA QT-NASBA was performed as described elsewhere. ${ }^{17}$ Briefly, nucleic acids were extracted from the blood samples using the guanidium isothiocyanate/silica procedure. ${ }^{25}$ QT-NASBA was performed on a NucliSens EasyQ analyser (bioMérieux, Boxtel, The Netherlands) using the NucliSens Basic Kit for amplification according to manufacturer's manual at a $\mathrm{KCl}$ concentration of $80 \mathrm{mmol} / \mathrm{L}$ in a total reaction volume of $10 \mu \mathrm{L}$ per reaction. The number of gametocytes was calculated in relation to a standard gametocyte stage $\mathrm{V}$ dilution series, using the time-point of amplification at which the fluorescence detecting target amplicons exceeded the mean fluorescence of three negative controls $+20 \mathrm{SD}$. A similar protocol was used for the $18 \mathrm{~S}$ rRNA QT-NASBA ${ }^{18}$ to confirm the presence of $P$. falciparum parasites.

Data analyses. Statistical analyses were carried out in SPSS version 12.0 and STATA 8.0. Pfs25 QT-NASBA gametocyte density was analyzed on a log-scale with negative samples included as zeros. The relation between gametocyte density and mosquito infection rates was fitted to linear, logistic, and exponential functions. The best results were presented based on $r^{2}$. For field samples, the relation between gametocyte density and oocyst counts in individual mosquitoes was determined using negative binomial models. Uninfected mosquitoes were included in these analyses, and a random effect was added to allow for the correlation between observations from the same individual. Regression coefficients $\beta$ with $95 \%$ confidence intervals (CIs) were presented. Proportions were compared using odds ratios (ORs) and the trend in proportions with the Cochran-Armitage test for trend. Normally distributed continuous variables were compared using the Stu- dent $t$ test, and the correlation between continuous normally distributed variables was quantified with the Pearson correlation coefficient.

\section{RESULTS}

Infectiousness of cultured gametocytes. The percentage of infected mosquitoes was positively associated with gametocyte density from in vitro culture $(\beta=6.1 ; 95 \% \mathrm{CI}, 2.7-9.6)$ and was described by a linear function $\left(r^{2}=0.39 ; P=0.001\right.$; Figure 1). Non-linear functions did not improve the fit. Infected mosquitoes harbored one to four oocysts, and only $14 \%$ of the infected mosquitoes had more than one oocyst, making analyses on oocyst counts uninformative. An estimated $7.6 \%$ of the feeding mosquitoes was infected at the microscopic detection limit of 5 gametocytes $/ \mu \mathrm{L}$. The trendline crossed the $y$-axis at 250-300 gametocytes $/ \mathrm{mL}$, indicating that mosquito infection under the used conditions is unlikely below this threshold. There was a good consensus between the gametocyte density measured in the Pfs25 QTNASBA and the estimated density from diluted starting concentrations (Pearson correlation coefficient: $0.88, P<0.001$ ).

Infectiousness of naturally infected children. A total of 100 membrane feedings were successfully carried out in children with a median age of 2.8 years (interquartile range, 2-4 years). At the time of membrane feedings, $25.0 \%$ (25/100) of the children harbored microscopically detectable gametocytes, and $75.0 \%(75 / 100)$ were gametocyte positive by $P f_{s} 25$ QTNASBA. A total of $62.0 \%(62 / 100)$ of the children were infectious to mosquitoes, of whom $69.4 \%$ (43/62) had no microscopically detectable gametocytes. There was a positive association between gametocyte density and the percentage of infected mosquitoes, described by an exponential function $\left(r^{2}=0.48 ; P=0.026\right.$; Figure 2). Infected mosquitoes har-

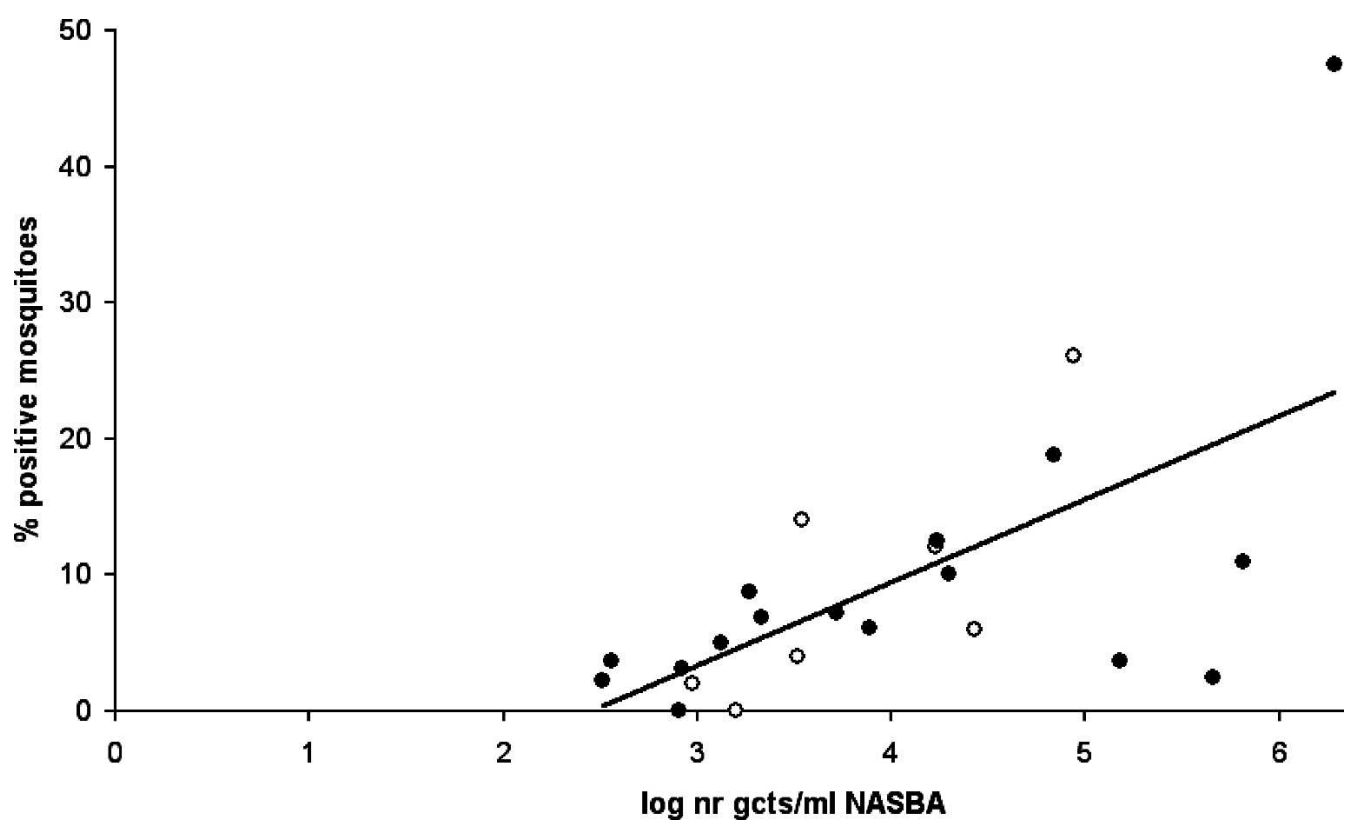

FIGURE 1. Mosquito infection rates against log gametocyte density for feeding experiments on serial dilutions of in vitro cultured $P$. falciparum gametocytes. Results are shown from three membrane feeding experiments with serial dilutions of non-synchronized $(\bigcirc)$ and synchronized $(\bullet)$ gametocytes. Gametocyte density was determined by $P f s 25$ QT-NASBA; mosquito infection rates were determined by examination of $50-100$ dissected mosquitoes per sample. The curve represents the linear relation between log gametocyte density and mosquito infection rates $(y=6.13 x$ $\left.-15.06 ; r^{2}=0.39, P=0.001\right)$. 


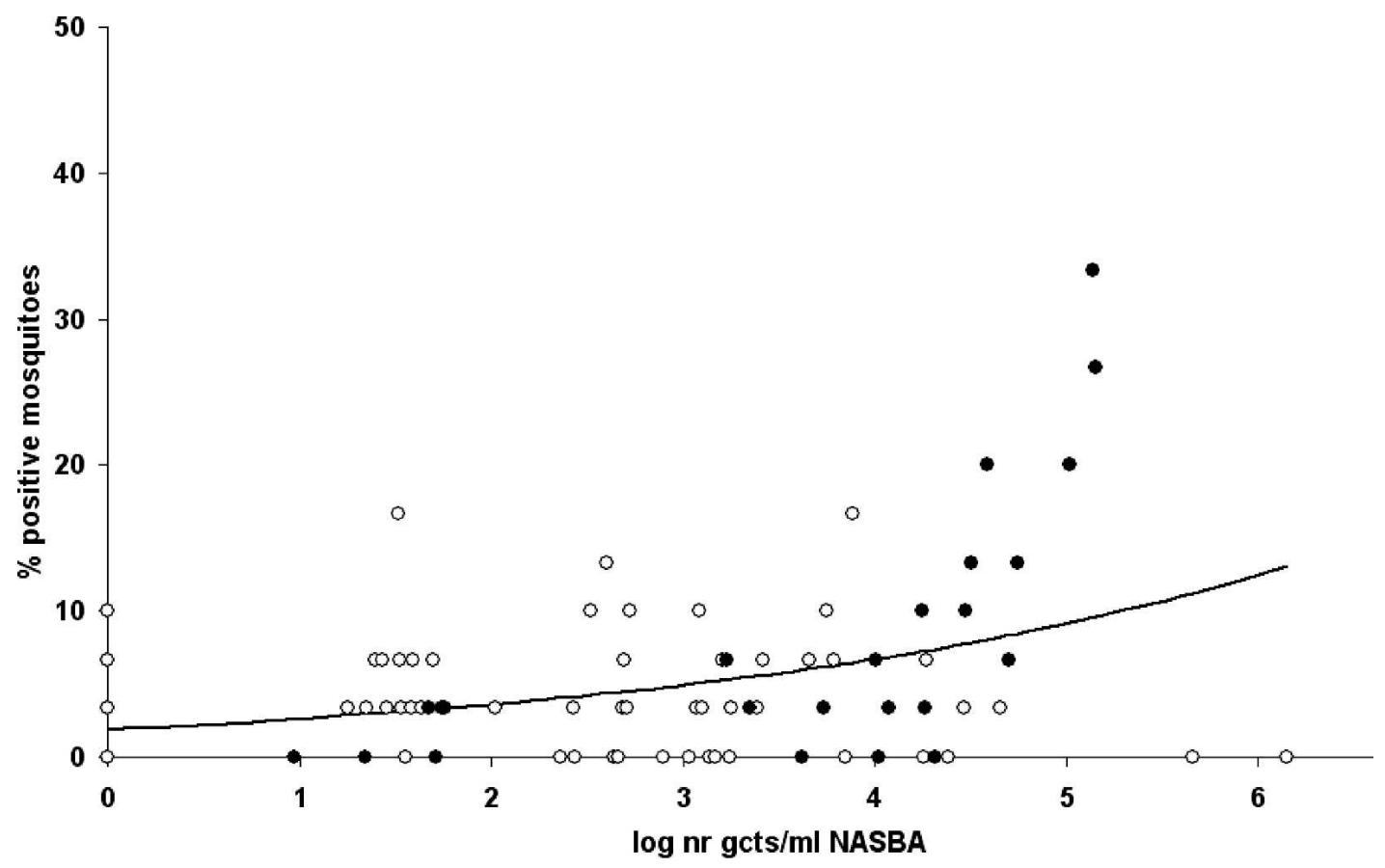

FiguRE 2. Mosquito infection rates against log gametocyte density for feeding experiments on venous blood samples of naturally infected Kenyan children. Results are shown for individual venous blood samples; microscopically confirmed gametocyte carriers are indicated with filled circles. Gametocyte density was determined by Pfs 25 QT-NASBA; mosquito infection rates were determined by examination of 30 dissected mosquitoes per sample. The curve represents the exponential relation between log gametocyte density and mosquito infection rates $(y=$ $\left.1.93 \mathrm{e}^{0.31 \mathrm{x}} ; r^{2}=0.48, P=0.026\right)$.

bored $1-13$ oocysts, and $26 \%$ of the infected mosquitoes had $>1$ oocyst. We observed a positive association between gametocyte density and oocyst counts in mosquitoes $(\beta=0.38$; 95\% CI, 0.14-0.61; $P=0.002$ ) and an independent borderline significant positive association between age and oocyst counts ( $\beta=0.11 ; 95 \% \mathrm{CI},-0.01$ to $0.23 ; P=0.075$ ). Eight children were infectious to mosquitoes with no gametocytes detected in their samples by $P f s 25$ QT-NASBA. P. falciparum $18 \mathrm{~S}$ ribosomal RNA was detected in all these samples (eight of eight), indicating that RNA extraction had been successful and $P$. falciparum parasites were present.

Infectiousness of children with submicroscopic gametocytemia. Successful mosquito infection was observed in children with microscopic and submicroscopic gametocyte densities (Table 1). Compared with children with microscopically detectable gametocytes, the proportion of infectious children was lower in children with submicroscopic gametocytemia and lowest in children who were negative in both microscopy and $P f s 25$ QT-NASBA (Cochran-Armitage test for trend, $P=0.001)$. The same trend was observed for the proportion of infected mosquitoes (Cochran-Armitage test for trend, $P<$ $0.001)$. Importantly, $70.0 \%(35 / 50)$ of the children with submicroscopic gametocytemia were capable of infecting at least one mosquito. Of those children who were gametocyte negative in both microscopy and $P f_{s} 25$ QT-NASBA, 32.0\% (8/25) were infectious to mosquitoes. In general, the association between gametocyte prevalence and the proportion of infectious children in the random feeds was weak for microscopy $(\mathrm{OR}=2.36 ; 95 \% \mathrm{CI}, 0.85-6.57)$ and much stronger for the Pfs25 QT-NASBA (OR $=5.46$; 95\% CI, 2.05-14.56). The contribution to malaria transmission of children with microscopically confirmed gametocytes and children with submi-

TABLE 1

Gametocyte carriage detected by microscopy and $P f_{s} 25$ QT-NASBA and infection of mosquitoes after membrane feeding

\begin{tabular}{|c|c|c|c|c|}
\hline & & Gametocyte carri & & $P$ \\
\hline Microscopy & - & - & + & \\
\hline Pfs25 QT-NASBA & - & + & + & \\
\hline Number of samples & 25 & 50 & 25 & \\
\hline Density, GM (IAR)* & - & $592.5(42.0-2,486.5)$ & $4,058.1(301.0-34,798.3)$ & $0.007 \dagger$ \\
\hline Infectious children, \% (N/N) & $32.0(8 / 25)$ & $70.0(35 / 50)$ & $76.0(19 / 25)$ & $0.001 \ddagger$ \\
\hline Infected mosquitoes, \% (N/N) & $1.7(13 / 750)$ & $4.1(62 / 1,500)$ & $8.3(62 / 750)$ & $<0.001$ \\
\hline Contribution to transmission, $\% \S$ & 9.5 & 45.3 & 45.3 & \\
\hline
\end{tabular}

Geometric mean density of gametocytes per $\mathrm{mL}$ of blood in the Pfs25 QT-NASBA for positives only.

$\uparrow P$ no value for the Student $t$ test on log-transformed gametocyte density.

$\ddagger P$ value for the Cochran-Armitage test for trends.

$\S$ The contribution of each group to transmission was estimated by dividing the number of mosquitoes infected by this group by the total number of mosquitoes infected by all 100 children. Equal mosquito exposure of all groups is assumed.

-, gametocyte negative; +, gametocyte positive; GM, geometric mean; IQR, interquartile range. 
croscopic gametocytemia was estimated by determining the proportion of the total number of infected mosquitoes that they infected. Although individual submicroscopic gametocyte carriers infected fewer mosquitoes, submicroscopic gametocytemia was more common than microscopic gametocytemia, resulting in an estimated contribution to transmission of $45.3 \%$ for both groups (Table 1 ).

\section{DISCUSSION}

In this study, we showed that submicroscopic gametocyte densities can efficiently infect mosquitoes under both laboratory and field conditions. Although it has previously been suggested that submicroscopic gametocyte densities can infect mosquitoes, ${ }^{3,6,8,9,12-14}$ this is the first study that actually quantifies submicroscopic gametocytemia in relation to mosquito infection. We found that the contribution of submicroscopic gametocytemia to malaria transmission can be similar to that of microscopically confirmed gametocyte carriers.

Data from cultured gametocytes showed a clear reduction in the proportion of infected mosquitoes with decreasing gametocyte density. Such a relation has been observed before ${ }^{24,26}$ but could not be extended to submicroscopic densities until recently. An estimated $7.6 \%$ of mosquitoes became infected after feeding on a sample with a gametocyte concentration at the threshold of microscopical detection. Successful mosquito infection becomes unlikely below an estimated density of 250-300 gametocytes/mL.

Under field conditions, submicroscopic gametocyte densities can also infect mosquitoes, although the association between gametocyte density and mosquito infection rates was less straightforward than in the laboratory experiments. A direct comparison between the experiments in the laboratory and the field can not be made for a number of reasons. There may be differences in gametocyte fitness or maturity between cultured NF54 gametocytes and natural infections, as well as differences in compatibility of parasite isolates and vector species. $^{27,28}$ Natural infections also regularly contain multiple parasite genotypes, opposed to a single infecting genotype in the in vitro culture. This could affect gametocyte development through in-host competition between parasite genotypes. ${ }^{29,30}$ Sexual stage-specific immune responses may also have influenced transmission efficiency in the field experiments. $^{31}$

In the field experiments, we frequently observed mosquito infection at very low gametocyte densities. Successful mosquito infection by eight children with negative Pfs25 QTNASBA results suggests that infection can even occur below the detection level of the $P f s 25$ QT-NASBA, which is 20-100 gametocytes $/ \mathrm{mL} .{ }^{18}$ Unlike for the laboratory experiments, we could not estimate a minimum gametocyte density for mosquito infection in the field. Gametocytes below levels of 100/ mL may not be detected by Pfs 25 QT-NASBA, and this may explain transmission without detection of $P f s 25$ mRNA. Findings from laboratory and field experiments indicate that the threshold gametocyte density necessary to infect mosquitoes is below the theoretical threshold of two gametocytes per mosquito blood meal $(2 \mu \mathrm{L})$. Because the uptake of gametocytes by mosquitoes follows a Poisson or negative binomial distribution, ${ }^{32}$ some successful infections can occur when feeding on samples with lower gametocyte densities. Several biologic mechanisms could facilitate successful mosquito in- fections at low gametocyte densities. Motility or aggregation of gametocytes may occur, favoring the encounter of males and females. ${ }^{32}$ This phenomenon could have influenced transmission efficiency in our experiments. Gametocytes may also preferentially accumulate in the subdermal capillaries. ${ }^{33,34}$ Although this could increase the transmission success of individuals with low peripheral gametocyte density under natural conditions, it will not have influenced our findings because we used the same venous blood samples for feeding experiments and gametocyte quantification.

Both laboratory and field experiments clearly show the efficient transmission of low-density gametocytemia, despite Anopheles immune responses that can substantially reduce transmission success. ${ }^{35}$ We observed a 2 -fold lower proportion of infected mosquitoes for children harboring submicroscopic gametocyte densities $(4.1 \%)$ compared with microscopic gametocyte densities $(8.3 \%)$. This lower proportion of infected mosquitoes was counterbalanced by the higher prevalence of submicroscopic gametocytemia in our population, resulting in a similar contribution to malaria transmission of carriers with microscopic and submicroscopic gametocytemia at the time we conducted our experiments. The duration of gametocyte carriage is obviously a crucial factor to determine the overall contribution of submicroscopic gametocytemia to malaria transmission, and this requires further research. Further studies should also determine the prevalence and infectiousness of submicroscopic gametocytemia in other populations and the possible relations with seasonality and transmission intensity.

Despite our selected population of children after antimalarial treatment, we feel that our findings are of public health importance. Interventions that aim to interrupt transmission will have to reduce gametocytemia to densities well below the microscopic detection limit and should also target carriers with submicroscopic gametocytes.

Received May 17, 2006. Accepted for publication October 25, 2006.

Acknowledgments: The authors thank Jolanda Klaassen, Astrid Pouwelsen, Laura Pelser, and Jacqueline van Haalen of Radboud University Nijmegen and Samuel Orao from ICIPE for help with mosquito dissections. The authors thank the community of Mbita and surrounding villages for their cooperation. The authors thank Steve Kaniaru, George Omweri, Nick Makio, Patrick Sawa, Bernard Kapesa, Kenneth Okoth, and Peter Ongele for work in the field.

Financial support: This work was supported by the Technology Foundation STW (Grant NFA6009) and WOTRO (WM 2003-00702).

Authors' addresses: Petra Schneider, Institute of Immunology and Infection Research, University of Edinburgh, Edinburgh, UK, E-mail: Petra.Schneider@ed.ac.uk. J. Teun Bousema, Marga van de Vegte-Bolmer, and Robert W. Sauerwein, Department of Medical Microbiology 268, Radboud University Nijmegen Medical Centre, PO Box 9101, 6500 HB, The Netherlands, Telephone: 31-243619515, E-mails: t.bousema@ncmls.ru.nl, M.vandeVegte@mmb.umcn.nl, and r.sauerwein@mmb.umcn.nl. Louis C. Gouagna, Institut de Recherche pour le Développement, 01 PO Box 182, Ouagadougou, Burkina Faso, E-mail: Louis-Clement.Gouagna@ird.bf. Silas Otieno, International Centre for Insect Physiology and Ecology, Mbita, PO Box 30772, Nairobi, Kenya, E-mail: silas_otieno@yahoo.co.uk. Sabah A. Omar, Kenya Medical Research Institute, PO Box 54840, Nairobi, Kenya, E-mail: OSabah@kemri.org.

\section{REFERENCES}

1. McKenzie FE, Jeffery GM, Collins WE, 2002. Plasmodium malariae infection boosts Plasmodium falciparum gametocyte production. Am J Trop Med Hyg 67: 411-414. 
2. Collins WE, Jeffery GM, 2003. A retrospective examination of mosquito infection on humans infected with Plasmodium falciparum. Am J Trop Med Hyg 68: 366-371.

3. Jeffery GM, Eyles DE, 1955. Infectivity to mosquitoes of Plasmodium falciparum as related to gametocyte density and duration of infection. Am J Trop Med Hyg 4: 781-789.

4. Tchuinkam T, Mulder B, Dechering K, Stoffels H, Verhave JP, Cot M, Carnevale P, Meuwissen JH, Robert V, 1993. Experimental infections of Anopheles gambiae with Plasmodium falciparum of naturally infected gametocyte carriers in Cameroon: Factors influencing the infectivity to mosquitoes. Trop Med Parasitol 44: 271-276.

5. Mulder B, Tchuinkam T, Dechering K, Verhave JP, Carnevale P, Meuwissen, RV, 1994. Malaria transmission-blocking activity in experimental infections of Anopheles gambiae from naturally infected Plasmodium falciparum gametocyte carriers. Trans R Soc Trop Med Hyg 88: 121-125.

6. Bonnet S, Gouagna C, Safeukui I, Meunier JY, Boudin C, 2000. Comparison of artificial membrane feeding with direct skin feeding to estimate infectiousness of Plasmodium falciparum gametocyte carriers to mosquitoes. Trans $R$ Soc Trop Med Hyg 94: 103-106.

7. Shute PG, Maryon M, 1951. A study of gametocytes in a West African strain of Plasmodium falciparum. Trans $R$ Soc Trop Med Hyg 44: 421-438.

8. Boudin C, Olivier M, Molez JF, Chiron JP, Ambroise-Thomas P, 1993. High human malarial infectivity to laboratory-bred Anopheles gambiae in a village in Burkina Faso. Am J Trop Med Hyg 48: 700-706.

9. Graves PM, 1980. Studies on the use of a membrane feeding technique for infecting Anopheles gambiae with Plasmodium falciparum. Trans R Soc Trop Med Hyg 74: 738-742.

10. Graves PM, Burkot TR, Carter R, Cattani JA, Lagog M, Parker J, Brabin BJ, Gibson FD, Bradley DJ, Alpers MP, 1988. Measurement of malarial infectivity of human populations to mosquitoes in the Madang area, Papua, New Guinea. Parasitology 96: 251-263.

11. Muirhead-Thomson RC, 1954. Factors determining the true reservoir of infection of Plasmodium falciparum and Wuchereria bacnrofti in a West African village. Trans R Soc Trop Med Hyg 48: 208-225.

12. Githeko AK, Brandling-Bennett AD, Beier M, Atieli F, Owaga M, Collins FH, 1992. The reservoir of Plasmodium falciparum malaria in a holoendemic area of western Kenya. Trans $R$ Soc Trop Med Hyg 86: 355-358.

13. Coleman RE, Kumpitak C, Ponlawat A, Maneechai N, Phunkitchar V, Rachapaew N, Zollner G, Sattabongkot J, 2004. Infectivity of asymptomatic Plasmodium-infected human populations to Anopheles dirus mosquitoes in western Thailand. $J$ Med Entomol 41: 201-208.

14. Gamage-Mendis AC, Rajakaruna J, Carter R, Mendis KN, 1991. Infectious reservoir of Plasmodium vivax and Plasmodium falciparum malaria in an endemic region of Sri Lanka. Am J Trop Med Hyg 45: 479-487.

15. Babiker A, Abdel-Wahab A, Ahmed S, Suleiman S, RanfordCartwright LC, Carter R, Walliker D, 1999. Detection of low level Plasmodium falciparum gametocytes using reverse transciptase polymerase chain reaction. Mol Biochem Parasitol 99: 143-148.

16. Menegon M, Severini C, Sannella A, Paglia MG, Sangare D, Abdel-Wahab, Abdel-Muhsin AA, Babiker H, Walliker D, Alano P, 2000. Genotyping of Plasmodium falciparum gametocytes by reverse transcriptase polymerase chain reaction. Mol Biochem Parasitol 111: 153-161.

17. Schneider P, Bousema T, Omar S, Gouagna L, Sawa P, Schallig H, Sauerwein R, 2006. (Sub)microscopic Plasmodium falciparum gametocytaemia in Kenyan children after treatment with sulphadoxine-pyrimethamine monotherapy or in combination with artesunate. Int J Parasitol 36: 403-408.
18. Schneider P, Schoone G, Schallig H, Verhage D, Telgt D, Eling W, Sauerwein R, 2004. Quantification of Plasmodium falciparum gametocytes in differential stages of development by quantitative nucleic acid sequence-based amplification. Mol Biochem Parasitol 137: 35-41.

19. Abdel-Wahab A, Abdel-Muhsin AM, Ali E, Suleiman S, Ahmed S, Walliker D, Babiker HA, 2002. Dynamics of gametocytes among Plasmodium falciparum clones in natural infections in an area of highly seasonal transmission. J Infect Dis 185: 18381842.

20. Nassir E, Abdel-Muhsin AM, Suliaman S, Kenyon F, Kheir A, Geha H, Ferguson HM, Walliker D, Babiker HA, 2005. Impact of genetic complexity on longevity and gametocytogenesis of Plasmodium falciparum during the dry and transmission-free season of eastern Sudan. Int J Parasitol 35: 49-55.

21. Bousema JT, Schneider P, Gouagna LC, Drakeley CJ, Tostmann A, Houben R, Githure JI, Ord R, Sutherland CJ, Omar SA, Sauerwein RW, 2006. Moderate effect of artemisinin-based combination therapy on transmission of Plasmodium falciparum. J Infect Dis 193: 1151-1159.

22. McKenzie FE, Bossert WH, 2005. An integrated model of Plasmodium falciparum dynamics. $J$ Theor Biol 232: 411-426.

23. Ponnudurai T, Lensen AH, Meis JF, Meuwissen JH, 1986. Synchronization of Plasmodium falciparum gametocytes using an automated suspension culture system. Parasitology 93: 263274.

24. Ponnudurai T, Lensen AH, Van Gemert GJ, Bensink MP, Bolmer M, Meuwissen JH, 1989. Infectivity of cultured Plasmodium falciparum gametocytes to mosquitoes. Parasitology 98: 165-173.

25. Boom R, Sol CJ, Salimans MM, Jansen CL, Wertheim-van Dillen PM, van der NJ, 1990. Rapid and simple method for purification of nucleic acids. J Clin Microbiol 28: 495-503.

26. Lensen A, Bril A, van den, V, Van Gemert GJ, Eling W, Sauerwein R, 1999. Plasmodium falciparum: Infectivity of cultured, synchronized gametocytes to mosquitoes. Exp Parasitol 91: 101-103.

27. Lambrechts L, Halbert J, Durand P, Gouagna LC, Koella JC, 2005. Host genotype by parasite genotype interactions underlying the resistance of anopheline mosquitoes to Plasmodium falciparum. Malar J 4: 3.

28. Vaughan JA, Noden BH, Beier JC, 1994. Sporogonic development of cultured Plasmodium falciparum in six species of laboratory-reared Anopheles mosquitoes. Am J Trop Med Hyg 51: 233-243.

29. McKenzie FE, Bossert WH, 1998. The optimal production of gametocytes by Plasmodium falciparum. J Theor Biol 193: 419-428.

30. Paul RE, Diallo M, Brey PT, 2004. Mosquitoes and transmission of malaria parasites - not just vectors. Malar J 3: 39.

31. Bousema JT, Drakeley CJ, Sauerwein RW, 2006. Sexual-stage antibody responses to $P$. falciparum in endemic populations. Curr Mol Med 6: 223-229.

32. Pichon G, Awono-Ambene HP, Robert V, 2000. High heterogeneity in the number of Plasmodium falciparum gametocytes in the bloodmeal of mosquitoes fed on the same host. Parasitology 121: 115-120.

33. Gautret P, Miltgen F, Gantier JC, Chabaud AG, Landau I, 1996. Enhanced gametocyte formation by Plasmodium chabaudi in immature erythrocytes: Pattern of production, sequestration, and infectivity to mosquitoes. J Parasitol 82: 900-906.

34. Talman AM, Domarle O, McKenzie FE, Ariey F, Robert V, 2004. Gametocytogenesis: The puberty of Plasmodium falciparum. Malar J 3: 24.

35. Whitten MM, Shiao SH, Levashina EA, 2006. Mosquito midguts and malaria: Cell biology, compartmentalization and immunology. Parasite Immunol 28: 121-130. 